\title{
Cardiopulmonary Response to Videogaming: Slaying Monsters Using Motion Sensor Versus Joystick Devices
}

\author{
Jeffrey D. Sherman, BS, Michael S. Sherman, MD, ${ }^{2}$ and Terry Heiman-Patterson, MD $^{3}$
}

\begin{abstract}
Objective: Replacing physical activity with videogaming has been implicated in causing obesity. Studies have shown that using motion-sensing controllers with activity-promoting videogames expends energy comparable to aerobic exercise; however, effects of motion-sensing controllers have not been examined with traditional (non-exercise-promoting) videogames.

Materials and Methods: We measured indirect calorimetry and heart rate in 14 subjects during rest and traditional videogaming using motion sensor and joystick controllers.

Results: Energy expenditure was higher while subjects were playing with the motion sensor $(1.30 \pm 0.32 \mathrm{kcal} /$ $\mathrm{kg} /$ hour $)$ than with the joystick $(1.07 \pm 0.26 \mathrm{kcal} / \mathrm{kg} /$ hour; $P<0.01)$ or resting $(0.91 \pm 0.24 \mathrm{kcal} / \mathrm{kg} / \mathrm{hour}$; $P<0.01$ ). Oxygen consumption during videogaming averaged 15.7 percent of predicted maximum for the motion sensor and 11.8 percent of maximum for the joystick. Minute ventilation was higher playing with the motion sensor $(10.7 \pm 3.5 \mathrm{~L} /$ minute $)$ than with the joystick $(8.6 \pm 1.8 \mathrm{~L} /$ minute; $P<0.02)$ or resting $(6.7 \pm 1.4 \mathrm{~L} /$ minute; $P<0.001)$, predominantly because of higher respiratory rates $(15.2 \pm 4.3$ versus $20.3 \pm 2.8$ versus $20.4 \pm 4.2$ beats/minute for resting, the joystick, and the motion sensor, respectively; $P<0.001$ ); tidal volume did not change significantly. Peak heart rate during gaming was 16.4 percent higher than resting $(78.0 \pm 12.0)$ for joystick $(90.1 \pm 15.0 ; P=0.002)$ and 17.4 percent higher for the motion sensor $(91.6 \pm 14.1 ; P=0.002)$; mean heart rate did not differ significantly.

Conclusions: Playing with a motion sensor burned significantly more calories than with a joystick, but the energy expended was modest. With both consoles, the increased respiratory rate without increasing tidal volume and the increased peak heart rate without increasing mean heart rate are consistent with psychological stimulation from videogaming, rather than a result of exercise. We conclude that using a motion sensor with traditional videogames does not provide adequate energy expenditure to provide cardiovascular conditioning.
\end{abstract}

\section{Introduction}

A N INCREASED PREVALENCE of obesity has been reported in adults and children in the United States and throughout the world. ${ }^{1}$ Replacement of outdoor physical activity with increased television viewing ${ }^{2}$ and console videogame use $^{3}$ have been implicated as contributing factors. Indeed, increased videogame use has been shown to correlate with increasing body mass index, ${ }^{2,4}$ blood pressure, and hyperlipidemia. ${ }^{5}$ Although studies of subjects playing videogames have shown an increase in energy expenditure over resting levels, the magnitude of the increase was reported to be lower than levels associated with standard physical exercise, below national recommendations for health maintenance, and below levels needed for cardiovascular conditioning. ${ }^{6}$

In recent years, Nintendo, Inc. (Kyoto, Japan), Sony (Tokyo, Japan), and Microsoft (Redmond, WA) have introduced console games that use a motion-sensing controller instead of a standard joystick controller. Game-related motion, such as swinging a bat or sword, turning a wheel, or throwing a ball, is input to the gaming system through movement of the controller, as opposed to the more limited joystick movement and button pressing performed in previous-generation systems. Although energy expenditure has been shown to increase to levels comparable to low-level

\footnotetext{
${ }^{1}$ California Institute of Technology, Pasadena, California.

${ }^{2}$ Division of Pulmonary and Critical Care Medicine and ${ }^{3}$ Department of Neurology, Drexel University College of Medicine, Philadelphia, Pennsylvania.
} 
exercise when subjects use motion-sensing devices to play games specifically designed to promote physical activity, ${ }^{6-12}$ it is not known whether the use of motion-sensing devices also increase energy expenditure when playing traditional adventure (e.g., "Legend of Zelda" [Nintendo]), first person shooter ("Halo" [Microsoft]), or platform ("Mario" [Nintendo]) games, which are more popular among videogamers (sales data as per vgchartz.com). We therefore measured the energy expended by subjects playing a prior-generation standard (non-exercise-promoting) videogame with a handheld button and joystick controller (Nintendo GameCube ${ }^{\mathrm{TM}}$ system) versus a motion sensor-based system (Nintendo $\mathrm{Wii}^{\mathrm{TM}}$ ) and compared the energy expended using both systems with the energy expended while resting.

The primary purpose of this study was to find out whether playing videogames with a motion sensor interface burns more calories than playing a videogame using a standard hand-held controller. Our secondary purpose was to characterize the cardiopulmonary exercise response to playing each system.

\section{Materials and Methods}

Subjects were recruited by advertising at regional high schools and at Drexel University College of Medicine (Philadelphia, PA). Subjects were asked to arrive at the laboratory at least 4 hours postprandially and avoid consuming caffeinated beverages for 8 hours prior to testing. Subjects were excluded if they had a history of cardiac or pulmonary disease, if they were pregnant, or if they had physical or physiologic impairments that would prevent their use of either videogame interface. Prior to beginning the study, subjects were familiarized with the videogame and the controllers until they understood the game process and were comfortable with both controls. Versions of "Legend of Zelda" for the Wii and GameCube were used for the study. This traditional adventure game was chosen because the same challenges were present on both console versions of the game and because the highintensity "battle" conditions of the game require frequent use of the game controllers. Each subject was started at the same level for each game. Subjects could choose to sit or stand during gameplay per their preference.

Resting energy expenditure (REE) was measured for 15 minutes using a metabolic cart (Ultima ${ }^{\mathrm{TM}}$ CardiO2; Medgraphics, St. Paul, MN). Subjects were asked to breathe normally while sitting still in a reclined chair while wearing a foam face mask fitted to a flow and gas sensor. Tidal volume, respiratory rate, minute ventilation, oxygen consumption $\left(\mathrm{VO}_{2}\right)$, carbon dioxide production $\left(\mathrm{VCO}_{2}\right)$, and heart rate were measured at 1-minute intervals. The $\mathrm{O}_{2}$ pulse was calculated by dividing the $\mathrm{VO}_{2}$ by the heart rate. The respiratory exchange ratio $(\mathrm{R})$ was calculated by dividing $\mathrm{VCO}_{2}$ by $\mathrm{VO}_{2}$. Caloric energy expenditure was calculated by the method of Weir. ${ }^{13}$ Comparison reference values for maximum exercise were per Puente-Maestu. ${ }^{14}$

Subjects were then asked to participate in two exercises in random order: (a) play for 15 minutes using the motion sensor system (Wii) or (b) play the same game for 15 minutes using the button/joystick controller (GameCube). Metabolic cart measurements were repeated during each videogame session as described above. During gameplay, $\mathrm{VO}_{2}$ and pulse were reported both as mean values averaged over the 15-minute session and as peak values. Peak $\mathrm{Vo}_{2}$ and peak heart rate were defined as the 1-minute interval with the highest values. Values for metabolic equivalents of task were calculated by dividing the average $\mathrm{VO}_{2}$ during videogame play by the subject's own resting $\mathrm{VO}_{2}$. Subjects were asked to rest for at least 15 minutes between exercises or until they were comfortable and ready for the next session at their baseline pulse rate (whichever was longer).

Results are given as mean \pm standard deviation values for each variable. Results among resting, motion sensor, and button/joystick controller sessions were compared by repeated-measures analysis of variance (ANOVA), and pairwise comparisons were made by paired Student's $t$ test. Results were considered significant at a $P$ value of $\leq 0.05$. Effect sizes for the $t$ tests were examined using the Cohen $d$, where a $d$ value of greater than 0.8 refers to a large effect size, a value of $0.5-0.8$ refers to a medium effect size, and a value of less than 0.5 refers to a small effect size.

Statistical analyses were completed using SPSS software (SPSS version 19; SPSS, Inc., Chicago, IL).

The study was approved by the Drexel University College of Medicine Institutional Review Board (protocol 17023). An informed consent form was signed by participants or, in the case of a minor, his or her parents. Assent forms were signed by participating minors.

\section{Results}

Fourteen subjects completed the study. Subject demographics are described in Table 1 . Subjects were all nonsmokers and varied in age from adolescence to middle age, in body mass index from underweight to obese, and in selfreported videogame experience from novice (never or rarely play videogames) to highly experienced (daily videogame play). All participants chose to sit while playing both videogames.

\section{Energy expenditure}

Playing with either the joystick or the motion sensor was associated with significant differences in energy expenditure compared with the subject's REE $(P<0.01$, ANOVA). Individual responses to videogame play are illustrated in Figure 1a. The mean REE for all subjects was $0.91 \pm 0.24 \mathrm{kcal} /$ $\mathrm{kg} /$ hour. Playing a videogame with the joystick controller resulted in an 18.8 percent increase over REE, increasing the energy expenditure to a mean of $1.07 \pm 0.26 \mathrm{kcal} / \mathrm{kg} / \mathrm{hour}$ $(P=0.02$, paired $t$ test; Cohen $d=0.67)$. Gaming with the

Table 1. Demographics of the Study Participants

\begin{tabular}{lc}
\hline Demographic & Value \\
\hline Age (years) & $31.2 \pm 14.6(17-58)$ \\
Sex (male/female) & $8 / 6$ \\
Weight $(\mathrm{kg})$ & $72 \pm 20$ \\
Body mass index $\left(\mathrm{kg} / \mathrm{m}^{2}\right)$ & $24.9 \pm 7.6(18.0-47.7)$ \\
Videogame experience & 6 \\
Experienced gamers & 3 \\
Occasional gaming & 5 \\
No experience & \\
\hline
\end{tabular}

Data are mean \pm SD values (range), mean \pm SD values, or number of subjects as indicated. 


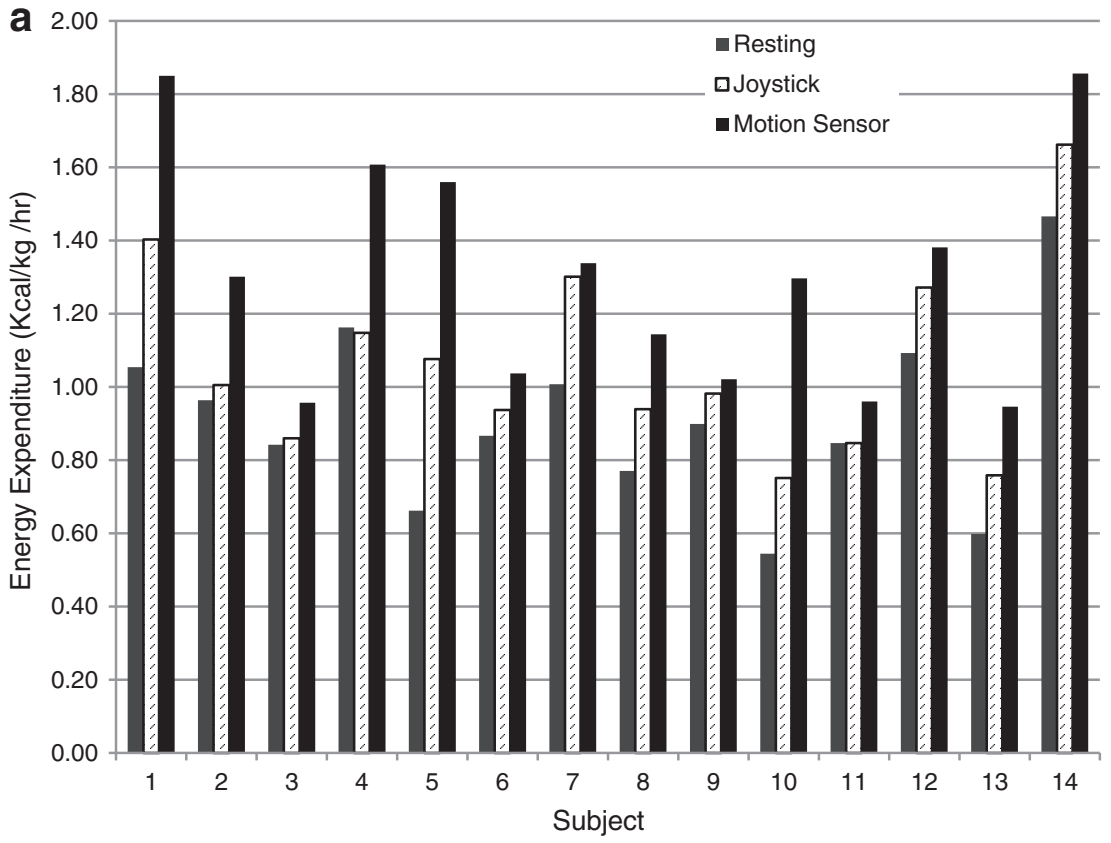

b

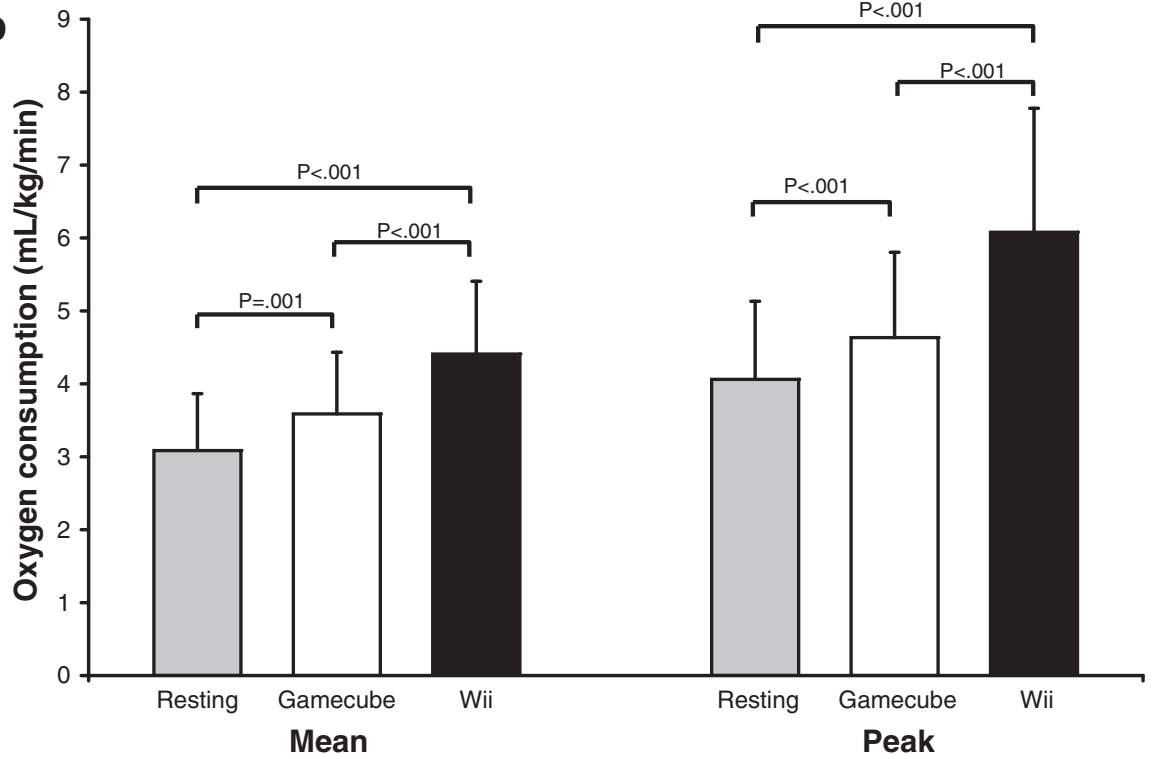

FIG. 1. Energy expenditure during videogame play versus resting: (a) individual energy expenditure measurements and (b) mean oxygen consumption among subjects resting, playing on the GameCube, and playing on the Wii. T-bars indicate standard deviation. motion-sensing controller further increased energy expenditure to a mean of $1.30 \pm 0.32 \mathrm{kcal} / \mathrm{kg} /$ hour, a 48.2 percent increase above REE $(P<0.01, t$ test; Cohen $d=1.38)$ and a $29.4 \%$ increase above playing with the joystick $(P<0.01, t$ test; Cohen $d=0.79$ ).

Similarly, mean $\mathrm{Vo}_{2}$ was significantly different among resting, joystick, and motion sensor play $(3.08 \pm 0.61 \mathrm{~mL} /$ minute $/ \mathrm{kg}$ versus $3.59 \pm 0.71 \mathrm{~mL} / \mathrm{minute} / \mathrm{kg}$ versus $4.41 \pm$ $1.14 \mathrm{~mL} / \mathrm{minute} / \mathrm{kg}$, respectively; $P<0.001$, repeated-measures ANOVA) (Fig. 1b), with an 18.3 percent higher $\mathrm{Vo}_{2}$ for the joystick versus REE $(P<0.01$; Cohen $d=0.77)$ and a 48.3 percent higher $\mathrm{VO}_{2}$ for the motion sensor versus $\operatorname{REE}(P<0.01$, Cohen $d=1.20)$. Playing with the motion sensor was associated with a 23.8 percent higher $\mathrm{VO}_{2}$ than playing using the joystick $(P<0.01$; Cohen $d=0.72)$.

Peak $\mathrm{VO}_{2}$ was also significantly different among resting, joystick, and motion sensor play $(P<0.001$, ANOVA) (Fig. 1b). The maximum $\mathrm{Vo}_{2}$ during rest was $4.06 \pm 1.07 \mathrm{~mL} /$ minute/kg. Playing with the joystick increased peak $\mathrm{VO}_{2}$ by 16.6 percent to $4.63 \pm 1.17 \mathrm{~mL} /$ minute $/ \mathrm{kg} \quad(P<0.02$; Cohen $d=0.51$ ), whereas playing the motion sensor device increased peak $\mathrm{VO}_{2}$ by 56.7 percent over resting to $6.59 \pm 1.5 \mathrm{~mL} / \mathrm{minute} / \mathrm{kg}(P<0.001$; Cohen $d=1.94)$ and by 32.68 percent over using the joystick $(P<0.001$; Cohen $d=1.45$ ). However, the subjects' average peak $\mathrm{Vo}_{2}$ while playing with the motion sensor was modest, at $19 \pm 9$ percent of predicted maximum $\mathrm{Vo}_{2}$. Subjects' average peak $\mathrm{VO}_{2}$ during joystick play was significantly lower than during motion sensor play, at $14 \pm 5$ percent of predicted maximum $\mathrm{VO}_{2}(P<0.01)$.

The average $\mathrm{R}$ value did not differ between activities $(0.89 \pm 0.06$ versus $0.91 \pm 0.07$ versus $0.89 \pm 0.06$ for resting, joystick, and motion sensor, respectively; $P=0.21$ ). Analysis of $\mathrm{V}$-slope plots $\left(\mathrm{VO}_{2}\right.$ versus $\left.\mathrm{VCO}_{2}\right)$ showed that no subject 
FIG. 2. Cardiac responses to videogame play: (a) individual peak heart rate and (b) mean $\mathrm{O}_{2}$ pulse among subjects resting, playing on the GameCube, and playing on the Wii. T-bars indicate standard deviation.
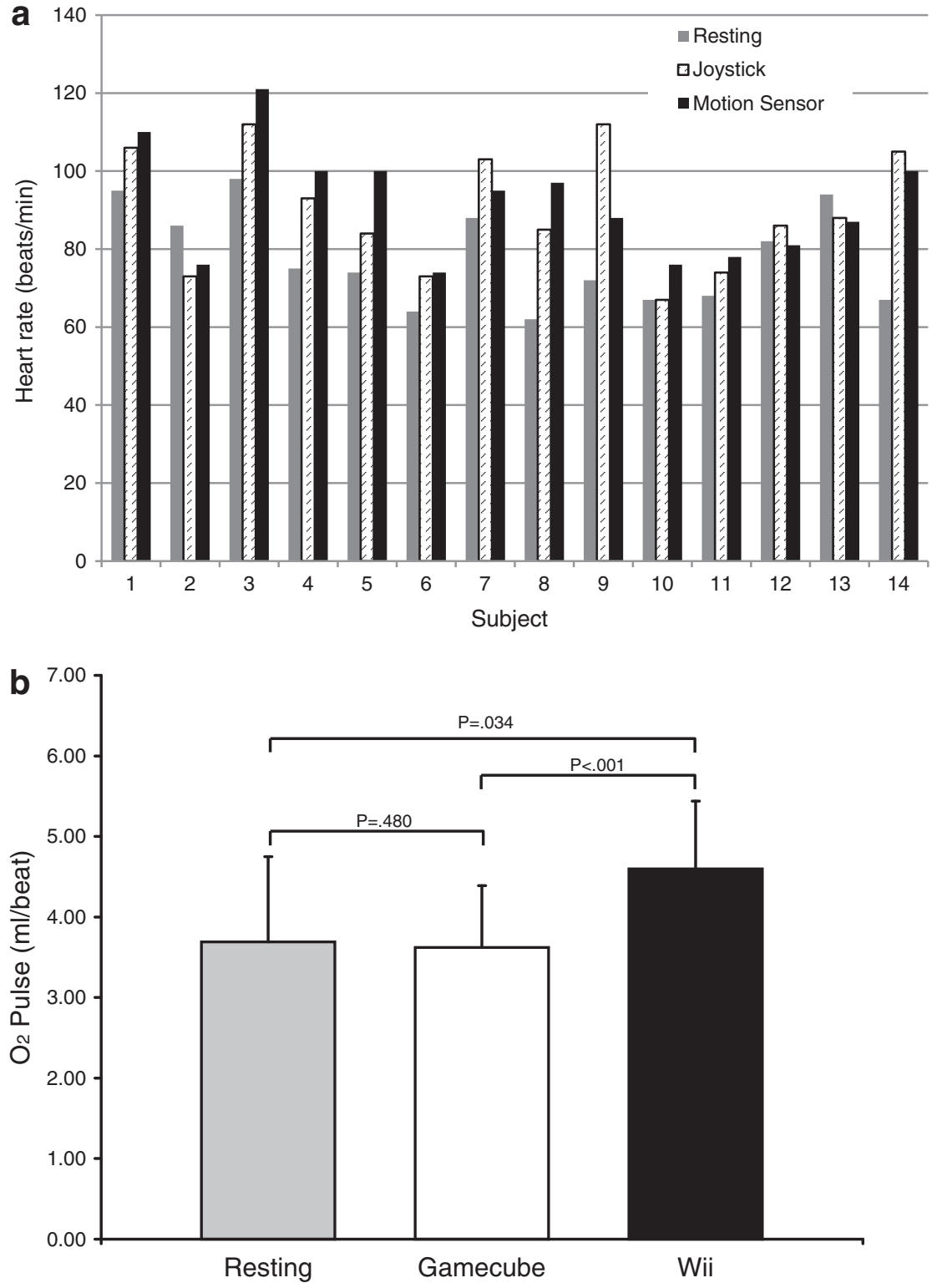

achieved anaerobic threshold during videogame play with either system.

\section{Cardiac response to videogame play}

Individual heart rate responses are illustrated in Figure 2a. Differences between maximum heart rates observed between resting and videogame play were statistically significant (78.0 \pm 12.0 versus $90.1 \pm 15.0$ versus $91.6 \pm 14.1$ beats/ minute for resting, joystick, and motion sensor, respectively; $P<0.01$, repeated-measures ANOVA). The difference in maximum heart rate between joystick and motion sensor play was not significant. The maximum heart rate achieved during joystick play was 16.3 percent higher than resting heart rate $(P=0.002$; Cohen $d=0.89)$, whereas using the motion sensor was associated with a maximum heart rate of 17.4 percent higher than resting ( $P=0.002$; Cohen $d=1.03$ ). However, these increases in heart rate were not sustained, as there was no significant difference in average heart rate over the course of each 15 -minute session $(77.9 \pm 12.2$ versus $82.3 \pm 15.0$ versus $83.6 \pm 14.0$ beats/minute for resting, joystick, and motion sensor, respectively; $P=$ not significant). At peak exercise, heart rate was $48 \pm 9$ percent versus $49 \pm 9$ percent of predicted maximum when using the joystick and motion sensor controllers, respectively.

There was no difference in peak $\mathrm{O}_{2}$ pulse between resting and playing using the joystick $(3.7 \pm 1.1 \mathrm{~mL} / \mathrm{beat}$ versus $3.6 \pm 0.8 \mathrm{~mL} /$ beat, respectively), but there was a 32.8 percent increase in $\mathrm{O}_{2}$ pulse between resting and the motion sensor $(3.7 \pm 1.1 \mathrm{~mL} /$ beat versus $4.6 \pm 0.8 \mathrm{~mL} /$ beat, respectively; $P<0.04$; Cohen's $d=0.95$ ) and a 29.4 percent increase between the joystick and the motion sensor $(3.6 \pm 0.8 \mathrm{~mL} / \mathrm{beat}$ versus $4.6 \pm 0.8 \mathrm{~mL} /$ beat, respectively; $P<0.001$; Cohen's $d=1.22$ ). Although statistically significant, the increased peak $\mathrm{O}_{2}$ pulse during motion sensor play was modest at $24.5 \pm 6.8$ percent of the predicted maximum $\mathrm{O}_{2}$ pulse (Fig. 2b). 


\section{Ventilatory response to videogame play}

Minute ventilation increased significantly during game play, predominantly owing to the increase in respiratory rate (Fig. 3a). Resting minute ventilation was $6.7 \pm 1.4 \mathrm{~L} /$ minute. During joystick game play, average minute ventilation increased 61 percent to $8.6 \pm 1.8 \mathrm{~L} /$ minute $(P<0.001$; Cohen's $d=1.20)$. Playing with the motion sensor increased the minute ventilation by 59 percent from resting, up to $10.7 \pm 3.5 \mathrm{~L} /$ minute $(P<0.001$; Cohen's $d=1.49)$. The difference in minute ventilation between joystick and motion sensor gameplay was 24 percent, which was also significant $(P<0.02$; Cohen's $d=0.76)$. The respiratory rate increased significantly during videogame play $(P<0.03$, ANOVA) (Fig. 3b). At rest, the average respiratory rate was $15.2 \pm 4.3$ breaths/minute. Videogame play on either system resulted in an almost identical increase in breathing rate over baseline: an increase of 41 percent to $20.3 \pm 2.8$ breaths/minute while playing with the joystick $(P<0.001$; Cohen's $d=1.40)$ and an increase of 43 percent to $20.4 \pm 4.2$ breaths/minute while playing with the motion sensor $(P=0.001$; Cohen's $d=1.22)$. There were no significant differences in tidal volume between resting and videogame play with either system.

\section{Discussion}

We found that energy expenditure was significantly higher when subjects were playing videogames than when resting and that energy expenditure was significantly higher when playing with the motion sensor versus playing with the joystick. However, the increase in energy expenditure with either videogame was modest in comparison with energy expended during other physical activities (Table 2). ${ }^{15} \mathrm{De}-$ spite the use of motion sensor technology, playing a traditional videogame with a motion sensor still fails to provide the level of exercise needed to promote cardiovascular
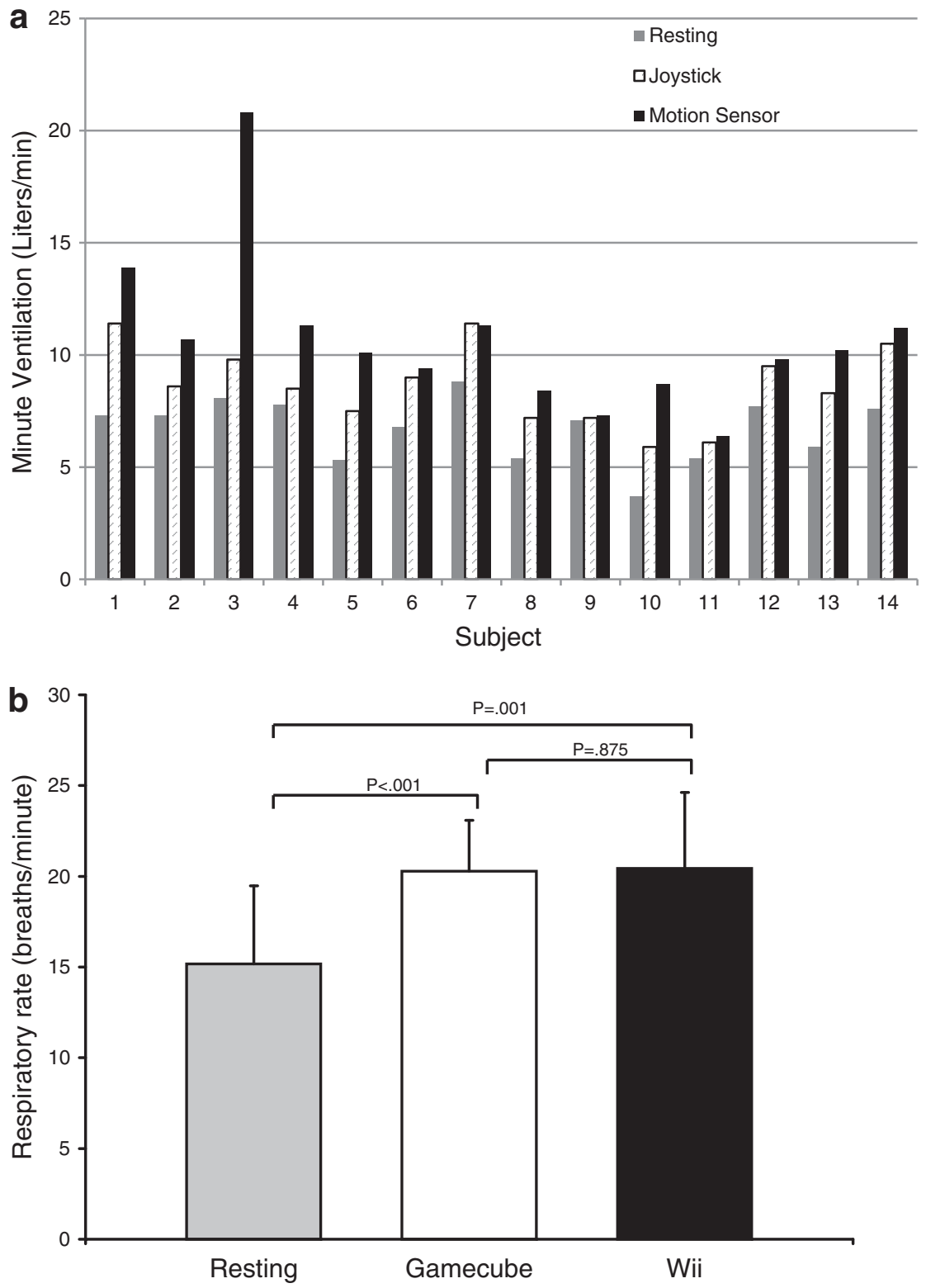

FIG. 3. Pulmonary responses to videogame play: (a) individual minute ventilation and (b) mean respiratory rate among subjects resting, playing on the GameCube, and playing on the Wii (Nintendo). $\mathrm{T}$-bars indicate standard deviation. 
Table 2. Comparison of Energy Expenditure of Videogaming With Physical Exercise and Activities ${ }^{15}$

\begin{tabular}{lc}
\hline Activity & METS \\
\hline Judo & 10.3 \\
Running (6 miles/hour) & 9.8 \\
Ultimate Frisbee & 8 \\
Jogging & 7 \\
Walking with crutches & 5 \\
Activity-promoting videogame, moderate effort & 3.8 \\
Walking, household & 2.0 \\
Drawing or writing & 1.8 \\
Watching a movie in a theater & 1.5 \\
Wii non-activity-promoting videogame & 1.4 \\
Sitting at a desk & 1.3 \\
GameCube & 1.2 \\
Resting & 1 \\
\hline
\end{tabular}

a“"Legend of Zelda" from Nintendo.

METS, metabolic equivalents of task.

conditioning. Indeed, the energy expenditure used when playing either videogame is similar to that used while doing desk work and pales in comparison to walking or other exercise. We found that the maximum increase seen with the motion sensor was only 19 percent of the predicted maximum exercise capacity. These findings are similar to the results of the study of Amonette et al., ${ }^{16}$ who also reported relatively small increases in energy expenditure with highand low-intensity videogaming when compared with maximum exercise. Based on our findings, we estimated that a 70$\mathrm{kg}$ male would burn off the calories in a standard 82-calorie chocolate chip cookie in approximately 66 minutes if playing with a joystick controller and in 54 minutes if playing with a motion sensor interface, compared with only 22 minutes for steady walking at a moderate pace of 3 miles/hour.

We found statistically significant increases in minute ventilation between rest and playing videogames with a joystick and between using the joystick and using the motion sensor. The respiratory rate increased significantly while playing with either the joystick or the motion sensor in relation to resting, but there was no difference between gaming systems. In contrast to other forms of exercise, we found that the increase in minute ventilation was due predominantly to the increase in respiratory rate; tidal volume did not increase during either joystick or motion sensor gameplay. The pattern of increased respiratory rate without change in tidal volume is similar to that previously described in models of anxiety ${ }^{17}$ and performance of mental arithmetic. ${ }^{18}$ In conjunction with the lack of a difference in respiratory rate between joystick and motion sensor play, this finding suggests that the respiratory response may be due to psychological stimulation from the game, rather than a response to exercise.

The cardiac response to gameplay was limited. Although the peak heart rate increased significantly between resting and use of either gaming system, this increase was not sustained throughout the exercise period, and we found no difference in mean heart rates during each activity. Moreover, the maximum heart rate peaked in the low 90 beats/minute for both games; at about 48 percent of the predicted value, this is well below the 80 percent predicted that has been suggested for promotion of fitness ${ }^{19}$ and may not meet
American College of Sports Medicine and American Heart Association recommendations for exercise (moderate exercise that "noticeably accelerates the heart rate" or vigorous intensity that causes a "substantial increase in heart rate"). ${ }^{20}$ The variability in heart rate during gameplay, along with the rapid return to resting levels while gameplay continued, suggests that the heart rate response may also be due to psychological stimulation from the game and not a response to exercise. Although there was a statistically significant increase in $\mathrm{O}_{2}$ pulse in subjects playing with the motion sensor that was not seen in subjects playing with the joystick interface, the increase was modest and not likely to provide a cardiovascular conditioning effect. Moreover, the $\mathrm{R}$ value stayed relatively constant at about 0.88 , suggesting that the anaerobic threshold was never reached. These findings are consistent with a very modest physiologic expenditure of the activity.

Our results showed that subjects playing a standard videogame on the Wii had substantially smaller increases in energy expenditure than the three- to fourfold increases in energy expenditure previously reported for playing "Wii Boxing", or "Wii Jogging.,"7,11 Our GameCube results showing an 18.2 percent increase in energy expenditure are somewhat lower than those reported by Wang and Perry, ${ }^{6}$ who found an increase in energy expenditure of about 50 percent in a younger group of children playing on a gaming device (PlayStation ${ }^{\mathrm{TM}}$ 2; Sony Computer Entertainment, Tokyo) with a similar controller. The lower increment found in our GameCube players may be due to a lower REE in our older population; additionally, the subjects in the study of Wang and Perry ${ }^{6}$ may have been more mobile and animated during gameplay owing to their younger ages as their baseline energy expenditure was reported to be high. Playing with the Wii was associated with a 48.2 percent increase in energy expenditure, which was similar to the increase reported by Wang and Perry. ${ }^{6}$

Our study is limited by the relatively small number of subjects. The increases in energy expenditure and $\mathrm{Vo}_{2}$ reported among resting and GameCube and Wii use were consistent among almost all subjects, however, and we therefore believe the possibility of a type I error is small. Because gameplay occurred in a clinical environment, it is also possible that we are underestimating the total caloric expenditure that occurs during videogaming in the home. Finally, our study was not powered to determine whether there was a difference between boys and girls, between obese and normal weight subjects, or between experienced and inexperienced gameplayers.

Overall, we found only a modest cardiopulmonary exercise response to playing standard videogames and a statistically significant but clinically inconsequential increase when subjects played a similar videogame using a motion control device. The energy expenditure from this type of videogame activity appears to be minimal and to be less than the energy expended by writing at a desk or playing croquet. Videogame activities of this type would not contribute to fitness and should not be considered as aerobic exercise. Videogame participants wishing to use videogame technology to provide a cardiovascular workout should be advised to use games that are specifically designed for exercise or to consider other methods to improve cardiovascular fitness or increase energy expenditure. In view of these findings, the 
study of Sit et al. ${ }^{21}$ is noteworthy in demonstrating a preference among young children for more active videogames when given a choice between active and more sedentary versions of the same game. This preference could translate into higher energy expenditures during real-life videogame play than we found in a laboratory setting.

\section{Acknowledgments}

The authors wish to thank the subjects of the study for their participation, the ALS Hope Foundation for the use of their metabolic cart, and Diana Winters for reviewing the manuscript and editorial assistance.

\section{Author Disclosure Statement}

No competing financial interests exist for any of the authors. The authors have no affiliation with Nintendo, which did not provide any funding and did not have any role in the design or conduct for this study.

M.S.S. had full access to all the data in the study and takes responsibility for the integrity of the data and the accuracy of the data analysis. All authors were responsible for study concept and design. J.D.S. and M.S.S. were responsible for acquisition of data. M.S.S. and T.H.-P. were responsible for analysis and interpretation of data. J.D.S. and M.S.S. were responsible for drafting of the manuscript. All authors were responsible for critical revision of the manuscript for important intellectual content. M.S.S. was responsible for statistical analysis. T.H.-P. was responsible for administrative, technical, or material support.

\section{References}

1. Ogden CL, Carroll MD, Curtin LR, et al. Prevalence of overweight and obesity in the United States, 1999-2004. JAMA 2006; 295:1549-1555.

2. Robinson TN. Television viewing and childhood obesity. Pediatr Clin North Am 2001; 48:1017-1025.

3. Tremblay MS, Willms JD. Is the Canadian childhood obesity epidemic related to physical inactivity? Int J Obes Relat Metab Disord 2003; 27:1100-1105.

4. Ballard M, Gray M, Reilly J, Noggle M. Correlates of video game screen time among males: Body mass, physical activity, and other media use. Eat Behav 2009; 10:161-167.

5. Goldfield GS, Kenny GP, Hadjiyannakis S, et al. Video game playing is independently associated with blood pressure and lipids in overweight and obese adolescents. PloS One 2011; 6:e26643.

6. Wang X, Perry AC. Metabolic and physiologic responses to video game play in 7- to 10-year-old boys. Arch Pediatr Adolesc Med 2006; 160:411-415.

7. Lanningham-Foster L, Foster RC, McCrady SK, et al. Activity-promoting video games and increased energy expenditure. J Pediatr 2009; 154:819-823.

8. Lanningham-Foster L, Jensen TB, Foster RC, et al. Energy expenditure of sedentary screen time compared with active screen time for children. Pediatrics 2006; 118:e1831-e1835.
9. Graf DL, Pratt LV, Hester CN, Short KR. Playing active video games increases energy expenditure in children. $\underline{\mathrm{Pe}-}$ diatrics 2009; 124:534-540.

10. Trost SG, Sundal D, Foster GD, et al. Effects of a pediatric weight management program with and without active video games: A randomized trial. JAMA Pediatr 2014; 168:407413.

11. O'Donovan C, Greally P, Canny G, et al. Active video games as an exercise tool for children with cystic fibrosis. J Cyst Fibros 2014; 13:341-346.

12. Xian Y, Kakinami L, Peterson ED, et al. Will Nintendo "Wii Fit" get you fit? An evaluation of the energy expenditure from active-play videogames. Games Health J 2014; 3:86-91.

13. Weir JB. New methods for calculating metabolic rate with special reference to protein metabolism. J Physiol 1949; 109:1-9.

14. Puente-Maestu L. Reference values in adults. In: Ward SA, Palange P, eds. Clinical Exercise Testing. European Respiratory Society Monograph 40. Lausanne, Switzerland: European Respiratory Society; 2007, pp. 165-185.

15. Ainsworth BE, Haskell WL, Herrmann SD, et al. The Compendium of Physical Activities Tracking Guide. Healthy Lifestyles Research Center, College of Nursing \& Health Innovation, Arizona State University. https://sites .google.com/site/compendiumofphysicalactivities (accessed December 20, 2011).

16. Amonette WE, Dupler TL, Stroud LC. Metabolic responses of upper-body accelerometer-controlled video games in adults. Appl Physiol Nutr Metab 2010; 35:643-649.

17. Masaoka Y, Homma I. The effect of anticipatory anxiety on breathing and metabolism in humans. Respir Physiol 2001; 128:171-177.

18. Mador MJ, Tobin MJ. Effect of alternations in mental activity on the breathing pattern in healthy subjects. Am Rev Respir Dis 1991; 144:481-487.

19. Baquet G, Van Praagh E, Berthoin S. Endurance training and aerobic fitness in young people. Sports Med 2003; 33:1127-1142.

20. Haskell WL, Lee IM, Pate RR, et al. Physical activity and public health: Updated recommendation for adults from the American College of Sports Medicine and the American Heart Association. Circulation 2007; 116:10811093.

21. Sit CHP, Lam JWK, McKenzie TL. Direct observation of children's preferences and activity levels during interactive and online electronic games. J Phys Act Health 2010; 7:484-489.

Address correspondence to: Michael S. Sherman, MD Division of Pulmonary and Critical Care Medicine Drexel University College of Medicine 245 North 15th Street, Mailstop 107 Philadelphia, PA 19102

E-mail: Msherman@drexelmed.edu 